\title{
Bimodal optical computers
}

\author{
H. John Caulfield, John H. Gruninger, Jacques E. Ludman, K. Steiglitz, H. Rabitz, J. Gelfand, and E. Tsoni
}

\begin{abstract}
Analog optical solutions of numerical problems tend to be fast, simple, and inaccurate. Digital optical or electronic solutions to the same problems tend to be slower, harder, and more accurate. In circumstances outlined here, hybrid analog-digital systems can be built which give the accuracy of digital solutions with intermediate degrees of speed and simplicity. Because at any instant these processors are working in either the analog or the digital mode, we call them bimodal optical computers.
\end{abstract}

\section{Introduction}

While optical digital computers have been drawing great attention, ${ }^{1-7}$ it is only in analog computation that optics is known to excel over electronics. In this paper we offer a limited exploration of a proposed link between these two fields of optics. That is, we will discuss hybrid optical numerical processors. We seek the numerical accuracy of digital computing while still retaining some of the speed and power advantages of analog optics. To do this we must mix analog optics with digital electronics (or electrooptics or optics) to bootstrap the accuracy. We call this hybrid a bimodal optical computer.

While some of these concepts are new to optics, many are not new to science in general. Our purpose in this paper is to call the attention of optics workers to this area. We will present a general approach and then specialize to one very specific and simple problem: Linear algebraic equations. The method is clearly extendable to nonlinear problems and other linear problems.

\section{Generic System}

The generic system is comprised of three properly interacting systems: an optical analog solver of the

H. J. Caulfield is with University of Alabama in Huntsville, Center for Applied Optics, Huntsville, Alabama 35899; J. H. Gruninger is with Aerodyne Research, Inc., 45 Manning Road, Billerica, Massachusetts 01821; J. E. Ludman is with Rome Air Development Center/ES, Hanscom AFB, Massachusetts 01731; E. Tsoni is with University of Crete, Department of Computer Science, Iraklion, Crete, Greece; the other authors are with Princeton University, Princeton, New Jersey 08544.

Received 6 May 1986.

0003-6935/86/183128-04\$02.00/0.

(C) 1986 Optical Society of America. basic problem; a memory; and an accurate (digital or hybrid) calculator of the solution accuracy. The basic cycle is as follows:

calculate an approximate solution with the optical analog processor; remember that solution to high accuracy; calculate the solution accuracy with the accurate computer;

repose the problem as an error reduction problem; solve with an optical analog processor;

using the just-calculated improvement and the stored prior solution, calculate and remember the improved solution with the accurate computer;

calculate the solution accuracy with the accurate computer;

if the solution is accurate enough, stop;

if not, recycle.

Clearly, the convergence condition is that the error be reduced in each iteration. If this is the case, as we will show, the optical analog processor no longer limits solution accuracy.

In a purely digital system, the primary consumer of space, weight, power, time, and cost would be the solver (direct or iterative) of the problem solved by the relatively small, low-weight, power conservative, fast, and inexpensive optical analog processor. Thus there is the potential for significant overall system improvement using this hybrid approach.

There are two major forms the accurate processor can take. First, it can be a special purpose, fast, inexpensive digital processor. For reasons which will soon become evident, we call the hybrid system involving such a processor a mathematical problem solver. Second, the accurate processor could be a physical system interacting with the world. The problem is then isomorphic with the control theory. We call such a processor a physical problem solver. With a mild effort, the reader should become convinced that these two problem solvers use the same mathematics. 


\section{Accuracy Analysis}

We will examine the bimodal optical computer (BOC) with specific emphasis on linear algebra as might be used, for example, for numerical solution of partial differential equations. The generic BOC method was originally proposed by Thompson ${ }^{6}$ some time ago for iteratively improving the precision of mechanical devices which were used for the simultaneous solution of linear equations. This method appears to provide some considerable benefit for situations where a low-accuracy but fast device is available for providing approximate solutions to partial differential equations. This can then be linked to a higher accuracy device which is particularly well suited for forward substitution of the approximate solution into the original equation. The BOC iterative scheme, besides having been proposed by Lord Kelvin, ${ }^{6}$ is a standard numerical approach to the iterative solution of linear systems and has solution of linear systems and has been analyzed with respect to numerical round-off error by Wilkinson ${ }^{7}$ and Stewart ${ }^{8}$ among others ${ }^{9}$. A working model of this analog and digital bimodal electrical computer has also been constructed by Karplus. ${ }^{10}$ This work reexplores and extends the prior work and incorporates modern linear and nonlinear optical computer techniques.

We can summarize this idea in the following way. Suppose we want to solve the $n$-dimensional linear system of equations,

$$
\mathrm{A} \mathbf{x}=\mathbf{b} .
$$

Here $A$ is a given matrix, $b$ is a given vector, and $\mathbf{x}$ is the sought-after solution vector.

These problems are of great interest in their own right. In addition such systems with high dimensions arise when linear partial differential equations are solved by the finite difference method. Many other problems can be recast in this form. Suppose further that we have built a discrete optical analog processor for this problem which gives an approximate solution that can be summarized with the equation

$$
\tilde{\mathrm{A}} \tilde{\mathbf{x}}=\tilde{\mathbf{b}},
$$

where $\tilde{\mathrm{A}}$ and $\tilde{\mathbf{b}}$ differ from $\mathrm{A}$ and $\mathbf{b}$ because of the limited accuracy of the analog components. We now have an approximate solution to our problem $\tilde{x}$, which typically is accurate to a few percent. Next, we use a digital electronic computer to form the residual

$$
\mathbf{r}=\mathbf{b}-\mathrm{A} \tilde{\mathbf{x}}
$$

using the actual high-precision versions of $A$ and $\mathbf{b}$. Notice that this step entails only substitution of the current solution $\mathbf{x}$ in the modal equations, a relatively fast operation for even a modest digital computer. Subtracting Eq. (3) from Eq. (1) with digital electronics, we can write

$$
A(\mathbf{x}-\tilde{\mathbf{x}})-\mathbf{r}=0
$$

call the current solution error

$$
\mathbf{x}-\tilde{\mathbf{x}}=\Delta \mathbf{x}
$$

and write Eq. (4) as

$$
\mathrm{A}(\Delta \mathrm{x})=\mathbf{r} .
$$

We now have a problem of the same form as the original with A being the same matrix, except with the inhomogeneity term $\mathbf{b}$ replaced by the residual vector r.

We now want to use the analog optical computer again to estimate $\Delta \mathbf{x}$ and refine the solution $\tilde{x}$, but we first scale the equations by an appropriate number $S$ to bring the voltages and currents back to the levels in the first solution. Thus we solve

$$
\tilde{\mathrm{A}} \mathbf{y}=S \mathbf{r}
$$

and then use the estimate

$$
\Delta \mathbf{x}=y / S
$$

to refine the current solution to

$$
\mathbf{x}=\tilde{\mathbf{x}}+\Delta \mathbf{x} .
$$

This process can be iterated and in favorable conditions will converge quickly to solutions of accuracy only by the digital computer representation of $\mathrm{A}, \mathrm{b}$, and the digital computation of Eq. (3). The description above for the iterative procedure was given in terms of a linear equation; however, this concept may also be applied to nonlinear systems and would take advantage of the unique capacity of nonlinear analog circuits for the solution of the nonlinear algebraic equations of the discretized system. An analysis similar to the above treatment will again apply since the equations become quasi-linear near the true solution.

We might call this a floating-point analog computation where the scaling parameter $S$ acts as a radix, varying from stage to stage with the size of the residuals in the equations. We note that this technique is quite similar to the very standard iterative numerical methods, such as Newton's method. In addition, we see that this technique marries analog and digital computers in a most congenial way-we take advantage of the speed and highly parallel nature of the analog system as well as the memory and high precision of the digital system in the external loop.

We have examined the stability and convergence properties of the iteration process for this BOC. To first order we can model the error caused by solving the system on an analog computer (Eq. 2) by [Ref. 8, Corollary (3.7)]

$$
(\mathrm{A}+\mathrm{E})^{-1}=(\mathrm{I}-\mathrm{F}) A^{-1},
$$

where $\mathrm{E}$ is the error in the matrix due to the analog representation. The norm of $\mathrm{F}$ is bounded by

$$
\|\mathbf{F}\| \leqslant \frac{k(\mathrm{~A})(\|\mathbf{E}\| /\|\mathbf{A}\|)}{1-[k(\mathrm{~A})\|\mathbf{E}\| /\|\mathbf{A}\|]} .
$$

$\|\cdot\|$ is a matrix norm, and the condition number of $A$ is defined by

$$
k(A)=\|\mathrm{A}\| \cdot\left\|\mathrm{A}^{-1}\right\| .
$$

Substituting Eq. 10 into Eq. 6 gives 


$$
\mathbf{x}_{k-1}=\mathbf{x}_{k}-\delta_{k}=\mathbf{x}_{k}-(\mathrm{I}-\mathrm{F}) \mathrm{A}^{-1}\left(\mathbf{b}-\mathrm{A} \mathbf{x}_{k}\right) .
$$

Letting $\mathbf{x}^{*}=\mathrm{A}^{-1} \mathbf{b}$ be the exact solution, we can rearrange this to yield

$$
\mathrm{x}_{k-1}-\mathrm{x}^{*}=-F\left(\mathrm{x}_{k}-\mathrm{x}^{*}\right),
$$

and taking the norms of both sides,

$$
\left\|\mathbf{x}_{k+1}-\mathbf{x}^{*}\right\|<\|\mathbf{F}\| \cdot\left\|\mathbf{x}_{k}-\mathbf{x}^{*}\right\| .
$$

We thus have a sufficient condition for geometric convergence of the process, namely, $\|F\|<1$, which is satisfied if

$$
k(\mathrm{~A})\left[\frac{\|\mathrm{E}\|}{\|\mathrm{A}\|}\right]<\frac{1}{2},
$$

where $k(\mathrm{~A})$ is the condition number of $\mathrm{A}$, and $\|\mathrm{E}\|$ is the error in the analog representation of the true matrix A. Of course, when convergence takes place, the errors in the digital computation may ultimately overtake the effect of the analog error that is modeled here, although the effects of analog noise may prevent that kind of ultimate accuracy.

Since $\|\mathrm{E}\| /\|\mathrm{A}\|$ may be $\sim 0.01$ for optical analog processors, Eq. (16) requires that $k(\mathrm{~A})<50$. This is quite restrictive but perhaps quite pessimistic. Simple equilibration of rows may change $A$ to $A^{\prime}$ with

$$
k(\mathrm{~A})^{\prime} \ll k(\mathrm{~A}) .
$$

Furthermore, a variety of other mathematical tricks can be performed. We can replace Eq. (9) with

$$
\mathbf{x}=\tilde{\mathbf{x}}+\theta \Delta \mathbf{x}
$$

and seek to use the convergence factor $\theta$ to force convergence in analogy with stochastic approximation. We can replace Eq. (6) by

$$
A+\mathbf{p q}^{T}(\Delta x)=\mathbf{r},
$$

where $\mathbf{q}$ is chosen orthogonal to $\Delta \mathbf{x}$ and $\mathbf{p}$ is a free vector so that $A$ and $\mathbf{p q}^{T}$ are of the same dimensionality as A. Calling

$$
\mathrm{A}^{\prime \prime}=\mathrm{A}+\mathbf{p q}^{T},
$$

we seek $\mathbf{p}$ values to make

$$
k\left(\mathbf{A}^{\prime \prime}\right) \ll k(\mathbf{A}) .
$$

\section{Nonlinear Problems}

Perhaps the most important payoff with BOCs may be associated with the solution of nonlinear problems. Many physical phenomena result in nonlinear differential or ultimately algebraic equations for solution. Such problems are notoriously difficult to treat by conventional numerical methods on digital computers. This comment follows since the algorithms will involve linearization or perhaps iteration with convergence being slow or perhaps nonexistent in highly nonlinear problems. A more suitable approach would be based on directly building the nonlinear behavior into the calculation process. It appears possible to construct hybrid machines based on this logic following lines parallel to that discussed in Sec. III. The key to this approach rests on the fact that nonlinear electronic or optical elements can be readily made and integrated together into an overall nonlinear computer.

As a simple example of a nonlinear problem we may consider the search for roots of a polynominal $p(x)$ in the real variable $x$. It is straightforward to use optical methods to evaluate polynomials via Horner's rule. Optical polynomial evaluation can be analog ${ }^{11}$ or digital. Some tricks to accommodate dynamic range, allow root searching by scanning, extend the range of problems addressed, etc. are given in the latter reference. Root searching for real roots simply by scanning through $x$ and watching for $p(x)=0$ conditions is straightforward and fast. It is, however, not likely to be highly accurate. Suppose we identify an approximate real root $x_{0}$. We can then evaluate $p\left(x_{0}\right)$ and

$$
p_{1}(x)=p(x)-p\left(x_{0}\right)
$$

digitally. Assuming we are now close to the true root, we can now change the scale of both $p_{1}$ and $x$ to gain sensitivity. We might substitute $y=10 x$ and $q_{1}=$ $10 p_{1}$ and then search $q_{1}(y)$ as before. This leads to a better approximation $x_{1}$ as can be verified by digital evaluation of $p\left(x_{1}\right)$. Accuracy is limited by the condition number of the polynomial because that limits the accuracy of the polynomial evaluation. Other similar examples can be found, and a general set of logic can be set forth as discussed below.

A nonlinear computer of the type discussed in the first paragraph could likely be of limited accuracy but capable of achieving an extremely rapid solution without the introduction of artificial linearization or iteration algorithms. The machine could be used alone or incorporated into an overall hybrid device along the lines discussed in Sec. III and the polynomial root searching example. This would entail introducing a high-accuracy digital computer as a means of monitoring residual errors. Updated corrections to the original fully nonlinear solution could be achieved by again using the nonlinear solution if it is close enough to the true answer that the nonlinear computer effectively operates in the linear mode after the first cycle. As an alternative it would be possible to construct an additional linearized version of the machine for the accuracy updates on the solution. These approaches may be theoretically modeled as well as demonstrated in the laboratory, and we plan to carry out such studies in the future.

\section{v. Conclusions}

Analog optics, when adequate for a task, is usually superior in speed, size, power consumption, and cost to all competitors. What we have suggested here is a means to extend the set of situations for which analog optics is adequate. Many studies remain to be performed on both algorithms and hardware. Nevertheless, the general concept of a hybrid system appears to be extremely promising.

Work sponsored primarily by the U.S. Army Research Office under contract DAAG-29-84-C-0026. 
Some of H. J. Caulfield's work was sponsored under SDI/IST prime contract N00014-85-K-0479.

\section{References}

1. H. J. Caulfield, J. A. Neff and W. T. Rhodes, "Optical Computing: The Coming Revolution in Optical Signal Processing," Laser Focus/Electro-Opt. 100-106, 108, 110 (Nov. 1983).

2. D. Psaltis, Ed., “Optical Computing," Special Issue of Opt. Eng. (Jan 1984).

3. H. J. Caulfield and H. Szu, "The Mutual Time-Frequency Content of Two Signals," Proc. IEEE 72, 902 (1984).

4. J. A. Neff, Ed., "Optical Computing," Special Issue of Opt. Eng. (Jan 1985).

5. H. J. Caulfield, R. Athale, and W. C. Collins, "Optical MatrixMatrix Multiplier Based on Outer Product Decomposition," Appl. Opt. 21, 2089 (1982); R. P. Bocker, H. J. Caulfield, and K. Bromley, "Rapid Unbiased Bipolar Incoherent Calculator Cube," Appl. Opt. 22, 804 (1983); P. S. Guilfoyle, "Rapid Unbiased Bipolar Incoherent Calculator Cube," Opt. Eng. 23, 20 (1984).

6. W. Thompson (Lord Kelvin), Proc. R. Soc. London 28, 111 (1878).

7. J. H. Wilkinson, Rounding Errors in Algebraic Processes (Prentice-Hall, Englewood Cliffs, NJ, 1964).

8. G. W. Stewart, Introduction to Matrix Computations (Academic, New York, 1973).

9. E. Tsoni, K. Steiglitz, J. Gelfand, and H. Rabitz, "Stability Second Convergence Properties of Lord Kelvin's Bimodal Computer," unpublished manuscript, Princeton U. Princeton, NJ, (Dec. 1984).

10. W. J. Karplus and R. A. Russell, "Increasing Digital Computer Efficiency with the aid of Error-Correcting Analog Subroutines," IEEE Trans. Comput. C-20, 831 (1971).

11. C. M. Verber, R. P. Kenan, H. J. Caulfield, J. E. Ludman, and D. P. Stilwell, "Pipelined Polynomial Processors Implemented with Integrated Optical Components," Appl. Opt. 23, 817 (1984).

\section{Patter continued from page 3121}

Optical monitoring of weld penetration

A system is being developed to monitor weld penetration optically and produce a signal for controlling an arc welder. The system is aimed at automatic welders, robot welders in particular. Made from small, low-cost components and utilizing optical fibers to conduct the signals, the system is immune to the electromagnetic interference that is common in industrial environments.

The monitor directs collimated light from a small diode laser at the molten pool of metal beneath the arc (see Fig. 16). A filter intercepts the reflected beam to suppress extraneous light, including light from the welding arc. A position-sensitive detector at a distance from the pool intercepts the beam reflected by the pool.

If the weld penetrates the workpiece completely, the curvature of the pool

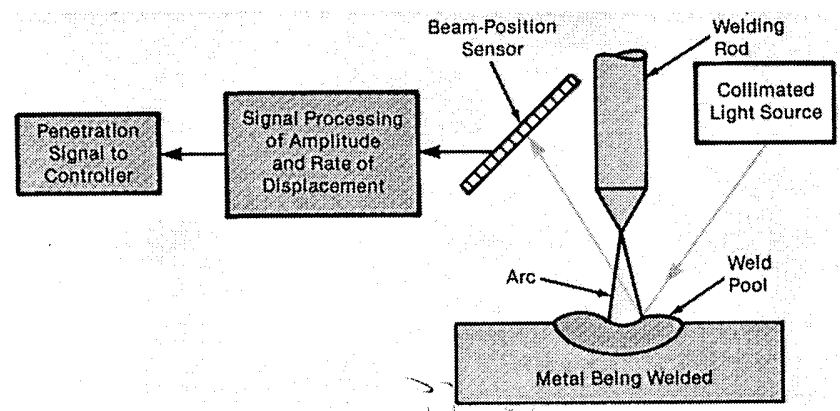

Fig. 16. Bounding off the meniscus of a pool of molten metal, a laser beam impinges on a position-sensitive photodetector. The beam diameter can be adjusted for the width of the weld. Optical filters screen out the light from the arc. surface suddenly changes. This causes a sudden deflection of the reflected light beam, and consequently a displacement of the beam spot on the detector. Signal-processing circuitry determines the amplitude and rate of beam dis placement to detect penetration and to generate control signals for the robot to regulate welding parameters.

The monitor is insensitive to changes in weld current, welder speed, and the thermal properties of the welded metal except as they affect weld penetration. The monitoring principle is adaptable to other types of welding, including tungsten/inert-gas, laser, and electron-beam techniques.

This work was done by Jonathan Maram of Rockwell International Corp. for Marshall Space Flight Center. Refer to MFS-29107.

\section{High-flux atomic-oxygen source}

A proposed apparatus can generate high fluxes (about $10^{15}$ atoms $/ \mathrm{cm}^{2}$-s) of ground state $\left({ }^{3} P\right)$ oxygen atoms. The kinetic energy would be variable in the range of 3-10 eV, and the beam would be free of contaminants, such as ions, metastable ${ }^{1} S$ or ${ }^{1} D$ oxygen atoms, or other neutral species. Designed specifically to study the degradation of materials and spacecraft glow phenomena in low earth orbits, this oxygen-atom beam source could be used to study gasphase collision phenomena involving energetic oxygen atoms.

In the proposed source (see Fig. 17) electrons are generated at a heated filament of $\mathrm{LaB}_{6}$ or $\mathrm{W}$. Bias voltages $V_{1}$ and $V_{2}$ accelerate the electrons to the proper energy $(6.5 \mathrm{eV})$ to maximize the dissociative attachment of a beam of $\mathrm{O}_{2}$ gas (that is, the separation of $\mathrm{O}_{2}$ molecules into $\mathrm{O}^{-}$ions). A solenoidal magnetic field provided by superconducting coils contains the electrons $e$ and the ions $\mathrm{O}^{-}$produced in the dissociative-attachment process.

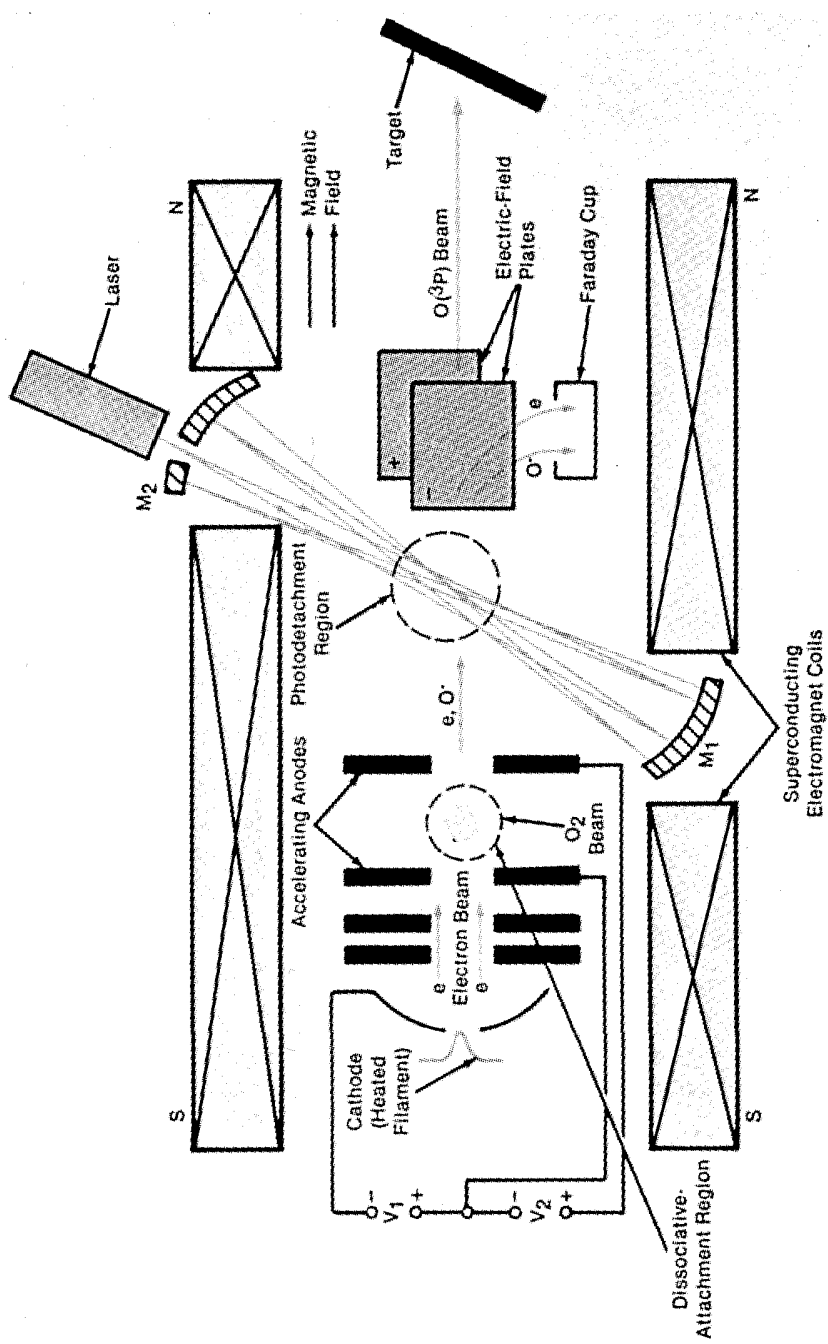

Fig. 17. Accelerated electrons strike a beam of $\mathrm{O}_{2}$ gas in the dissociative-attachment region, producing $\mathrm{O}^{-}$ions. The $\mathrm{O}^{-}$ions are accelerated to the desired final energy and pass through the photodetachment region to form $\mathrm{O}\left({ }^{3} P\right)$ atoms. These pass between electric field plates to remove $\mathrm{O}^{-}$and $e$ and then strike the target. continued on page 3268 